\title{
Preparation, characterization and in vitro drug release of simvastatin loaded PLGA microspheres
}

Pinto NPJ, Bazzo GC, Aragones A, Magini RS.

Federal University of Santa Catarina - Florianopolis, Brazil.

\section{Background and Aim}

Background: The use of simvastatin (SIM) associated with conducted materials, which control their release has been tested in several studies, and PLGA is one of that. PLGA microsphere with simvastatin has shown efficacy in the release not to be toxic, besides it promotes the cellular aggregation and proliferation in the bone tissue, nevertheless the use of this material form is scant without their incorporation to scaffolds.

Aim: The aim of this study was to characterize the PLGA microspheres encapsulating simvastatin, concentration of drug encapsulated, and in vitro drug release in different periods to obtain a novel biomaterial for bone regeneration, composed of free microspheres without scaffolds.

\section{Materials and Methods}

The microspheres of PLGA (82:18) with simvastatin were prepared through the $\mathrm{O} / \mathrm{W}$ emulsion solvent evaporation technique, in a drug polymer ratio of $1: 10$. The morphology of microspheres was analyzed by scanning electron microscopy. Micro particle size was determined by laser diffraction. The SIM incorporated to microspheres were characterized by reflectance Fourier transform spectroscopy (FT-IR). The drug encapsulation efficacy was evaluated in duplicate to determine the amount of SIM that was successfully loaded into the PLGA microspheres, using a UV spectrophotometer at wavelength $240 \mathrm{~nm}$. The sample was incubated in PBS $\left(\mathrm{pH} \mathrm{7,4)}\right.$, and immersed in a thermostatic bath at $36^{\circ} \mathrm{C}$ for 25 days to determine the in vitro drug release, it was analyzed in triplicate.

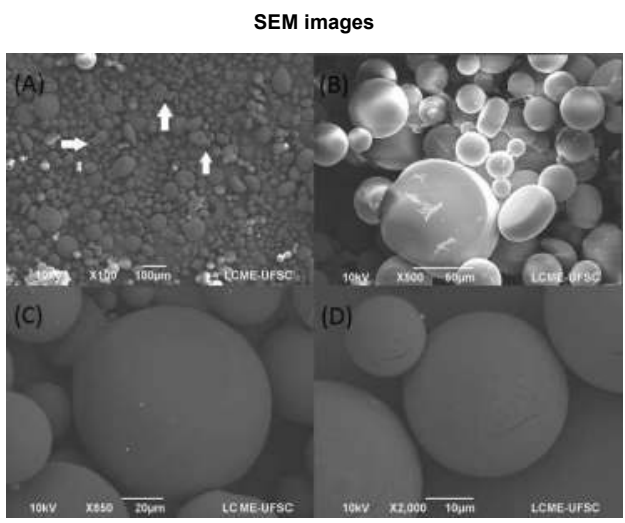

Photomicrografies of microparticles obtained by SEM, in magnification of (A) 100x, (B) 500x, (C) 850x and (D) 2000x. The arrows indicating some coalescence and agglomeration.

FT-IR
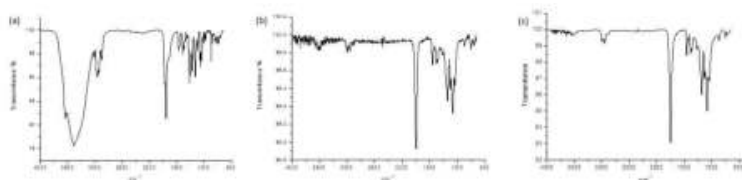

FT-IR spectra from SIM (a), PLGA (b) and SIM loaded PLGA microspheres (c).

\section{Results}

The O/W emulsion solvent evaporation method to prepare SIM loaded PLGA microspheres obtained good results. The morphology of microspheres was homogeneous with smooth surfaces, and regular spherical shape. The size was widely distributed, $80 \%$ was between 37 $\mu \mathrm{m}-690 \mu \mathrm{m}$. The drug encapsulation efficacy was $85.2 \%( \pm 3.53)$ in $10 \%$ drug to polymer ratio, and the FT-IR spectrum confirmed that SIM was successfully loaded into the polymer. In vitro study demonstrated a slow release of SIM during 25 days, only $5 \%$ of the drug was release.

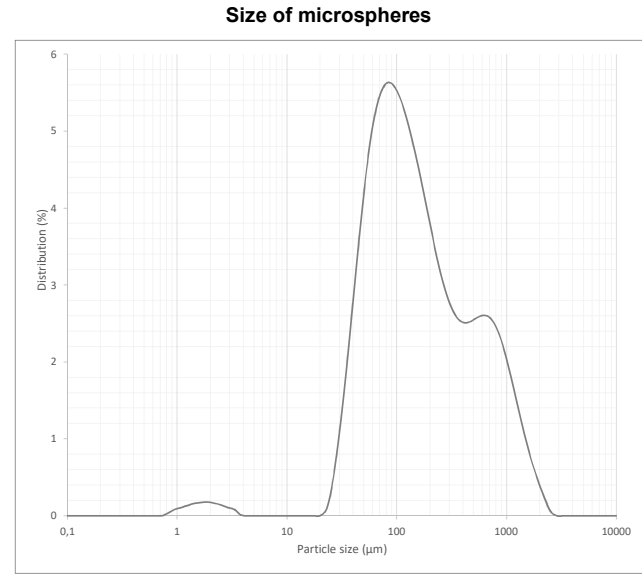

Distribution of microspheres particle size analysis.

In vitro SIM release

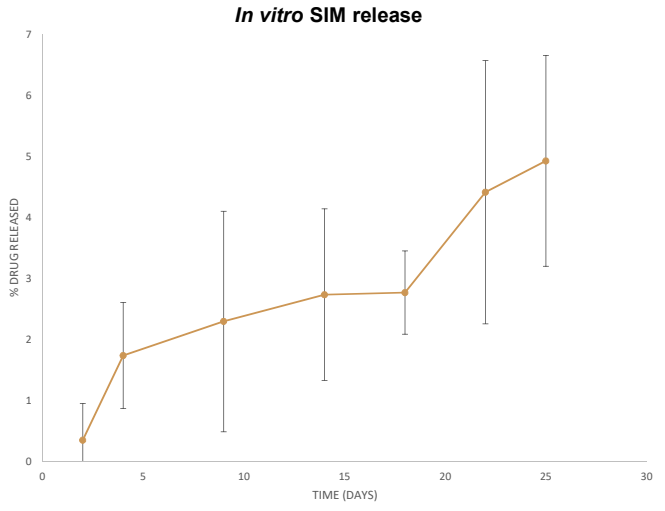

SIM drug release profile at 25 days in PBS.

\section{Conclusions}

These data suggest that drug-loaded microspheres has potential to be use as a biomaterial for enhancing bone formation, due its capacity to provide effective controlled release of SIM, and possibly decrease the tissue inflammatory response occurred in high values of released SIM.

\section{References} 1. Bao TQ, Hiep NT, Kim YH, Yang HM, Lee BT. Fabrication and characterization of
porous poly(lactic-co-glycolic acid) (PLGA) microspheres for use as a drug delivery Nath SD, Linh NT, Sadiasa A, Lee BT. Encapsulation of simvastatin in PLGA
microspheres loaded into hydrogel loaded BCP porous spongy scaffold as a
controlled drug delivery system for bone tissue regeneration. microspheres loaded into hydrogel loaded BCP porous spongy scaffold as a
controlled drug delivery system for bone tissue regeneration. J Biomater Appl.
2014;28(8):1151-63.

Naito Y, Terukina T, Galli S, Kozai Y, Vandeweghe S, Tagami T, Ozeki T, Ichikawa T critical size bone defect in the rabbit calvaria. Int J Pharm. 2014;461:157-62.

4. Gentile P, Nandagiri VK, Daly J, Chiono V, Mattu C, Tonda-Turo C, Ciardelli G Ramtoola Z Localised controlled release of simvastatin from porous chitosangelatin scaffolds engrafted with simvastatin loaded PLGA-microparticles for bone 